\title{
BMJ Open Alterations in the brain's connectome during recovery from severe traumatic brain injury: protocol for a longitudinal prospective study
}

\author{
Virginia Conde, ${ }^{1}$ Sara Hesby Andreasen, ${ }^{1,2}$ Tue Hvass Petersen, ${ }^{2}$ \\ Karen Busted Larsen, ${ }^{2}$ Karine Madsen, ${ }^{3}$ Kasper Winther Andersen, ${ }^{1}$ Irina Akopian, ${ }^{1}$ \\ Kristoffer Hougaard Madsen, ${ }^{1,4}$ Christian Pilebæk Hansen, ${ }^{2}$ Ingrid Poulsen, ${ }^{2}$ \\ Lars Peter Kammersgaard, ${ }^{2}$ Hartwig Roman Siebner ${ }^{1,5}$
}

To cite: Conde V, Andreasen SH, Petersen TH, et al. Alterations in the brain's connectome during recovery from severe traumatic brain injury: protocol for a longitudinal prospective study. BMJ Open 2017;7:e016286. doi:10.1136/ bmjopen-2017-016286

- Prepublication history for this paper is available online. To view these files please visit the journal online (http://dx.doi org/10.1136/bmjopen-2017016286)

Received 11 February 2017 Revised 26 April 2017 Accepted 3 May 2017

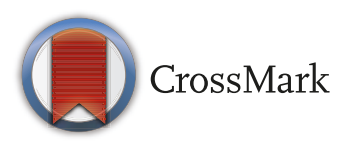

For numbered affiliations see end of article.

Correspondence to Hartwig Roman Siebner; h.siebner@drcmr.dk

\section{ABSTRACT}

Introduction Traumatic brain injury (TBI) is considered one of the most pervasive causes of disability in people under the age of 45. TBI often results in disorders of consciousness, and clinical assessment of the state of consciousness in these patients is challenging due to the lack of behavioural responsiveness. Functional neuroimaging offers a means to assess these patients without the need for behavioural signs, indicating that brain connectivity plays a major role in consciousness emergence and maintenance. However, little is known regarding how changes in connectivity during recovery from TBI accompany changes in the level of consciousness. Here, we aim to combine cutting-edge neuroimaging techniques to follow changes in brain connectivity in patients recovering from severe TBI. Methods and analysis A multimodal, longitudinal assessment of 30 patients in the subacute stage after severe TBI will be made comprising an MRI session combined with electroencephalography (EEG), a positron emission tomography session and a transcranial magnetic stimulation (TMS) combined with EEG (TMS/EEG) session. A group of 20 healthy participants will be included for comparison. Four sessions for patients and two sessions for healthy participants will be planned. Data analysis techniques will focus on whole-brain, both data-driven and hypothesis-driven, connectivity measures that will be specific to the imaging modality.

Ethics and dissemination The project has received ethical approval by the local ethics committee of the Capital Region of Denmark and by the Danish Data Protection. Results will be published as original research articles in peer-reviewed journals and disseminated in international conferences. None of the measurements will have any direct clinical impact on the patients included in the study but may benefit future patients through a better understanding of the mechanisms underlying the recovery process after TBI.

Trial registration number: NCT02424656; Pre-results.

\section{INTRODUCTION}

According to the International Initiative for Traumatic Brain Injury Research, TBI
Strengths and limitations of this study

- Employing a unique combination of cuttingedge neuroimaging techniques, the study will prospectively assess changes in the brain's functional and structural connectome related to loss of consciousness after severe traumatic brain injury (TBI).

- The repeated assessment of functional and structural brain connectivity over the course of 1 year will reveal plastic changes in the brain's connectome that accompany clinical changes in consciousness level, providing the potential to identify reliable biomarkers of recovery from impaired consciousness.

- A relatively large sample of patients in a subacute stage recovering from severe TBI will be studied. Considering the heterogeneity of the population of patients with TBI in regards to structural brain damage, we expect a diverse group of patients with different lesion maps.

- Given the longitudinal nature of the study design, not all patients included will have a full dataset mostly due to potential withdrawal from the study, death or exclusion criteria that arise during recovery.

- Different patients may have different rehabilitation programmes and different medications, and this observational study will not control for or interfere with the clinical therapies provided at the rehabilitation ward.

is considered one of the most pervasive causes of disability in people under the age of 45 with an incidence of approximately $500 / 100.000$ in Europe mostly due to road traffic accidents. ${ }^{1}$ At present, disorders of consciousness (DOC) derived from TBI are identified based on the duration of post-traumatic amnesia (PTA) and duration of loss of consciousness (LOC) after brain injury, which are considered the most 
reliable predictors of long-term prognosis following brain injury. ${ }^{23}$ However, PTA and duration of LOC are not useful as prognostic tools in the early stages after severe TBI, because information on length of amnesia and unconsciousness are not fully assessed until patients emerge from said conditions. ${ }^{23}$ Thus, appropriate diagnosis and in turn prognosis remain challenging for as long as they are based on behavioural signs of consciousness from the patients.

Over the past 15 years, functional brain mapping techniques have greatly expanded our knowledge on residual brain function after severe TBI. ${ }^{4}$ In particular, functional MRI (fMRI) studies have shown that some patients with severe brain injury but without behavioural signs of consciousness were able to display residual cortical processing related to aspects of speech perception and even conscious awareness, indicating a potential for recovery that otherwise would have remained unrecognised. ${ }^{56}$ As a consequence, fMRI as well as other functional brain mapping techniques have attracted considerable interest as a possible tool for aiding diagnosis and prognosis particularly in patients with severe TBI and DOC. ${ }^{7}$ In addition to functional brain mapping, $\left[{ }^{18} \mathrm{~F}\right]$-fluorodeoxyglucose positron emission tomography (FDG-PET), which is used to estimate the cerebral metabolic rate of glucose (CMRglc), has been used in this patient population. By the use of FDG-PET, patterns of cerebral hypometabolism have been identified in TBI patients with varying levels of consciousness, indicating that such areas are dysfunctional or dormant and that synaptic activity is below normal levels. Moreover, these patterns seem to be different between clinical states associated with DOC. ${ }^{8-10}$ Such patterns of glucose metabolism within patients have shown unprecedented high rates of correct prognosis. ${ }^{811}$

Patients who survive severe TBI can show a transition from coma towards different clinical conditions that are associated with various levels of consciousness, ${ }^{12}$ from being awake but not aware of oneself and the surroundings (vegetative state (VS)/unresponsive wakefulness syndrome (UWS) ) to being awake and minimally (and not consistently) aware (minimally conscious state (MCS)). Patients can fully recover consciousness and may signal it by functionally interacting with the environment, which is referred to as emergence from MCS. ${ }^{13}$ While the prognosis for patients who remain in coma is uniformly poor, prognosis for patients admitted to in-hospital rehabilitation with VS/UWS is somewhat better, and some recovery of function for patients in MCS after TBI is certainly possible. ${ }^{14}$ This is especially true in the case of patients regaining consciousness within a few weeks after injury. ${ }^{15}$ At present, misdiagnosis between UWS/VS and MCS can reach levels as high as $40 \%$ with standard clinical testing, which ultimately affects the clinical prognosis and rehabilitation programmes. ${ }^{16}$ Strikingly, the brain connectivity changes that occur during recovery and the underlying mechanisms that result in either the emergence from or a persistent DOC are at present not understood, and no reliable biomarkers that predict recovery have yet been identified.

Two influential models define consciousness as an emergent property of the integrated behaviour of widespread cortical networks. The information integration theory of consciousness states that consciousness is secured by a large repertoire of available states in which the brain can integrate information. ${ }^{17}$ According to the global neuronal workspace theory of consciousness, conscious access occurs when incoming information is made globally available to multiple brain systems through longrange cortico-cortical neuronal connections between prefrontal, parieto-temporal and cingulate cortices. ${ }^{18}$ Accordingly, electroencephalography (EEG) and fMRI studies have consistently shown a degradation of longrange cortico-cortical connectivity in unconscious brain states such as sleep, anaesthesia or TBI. ${ }^{19-21}$

Building on previous TBI neuroimaging research, the overall aim of this longitudinal multimodal trial is to prospectively assess dynamics of cortico-cortical brain connectivity changes after TBI and to derive patterns of metrics that probe connectivity. For this purpose, a range of advanced brain mapping techniques, which do not rely on the patient's ability to produce behavioural responses, will be applied. Using a prospective and longitudinal study design, repeated assessment of the brain's connectivity during the subacute stage (ie, period of specialised neurorehabilitation after discharge from neurointensive care) and at 1-year follow-up will be performed. The project will aim to include the largest group of TBI patients $(n=30)$ in the subacute stage studied so far with multiple neuroimaging modalities. Finally, strict inclusion criteria and comprehensive clinical and neuroradiological assessments will be performed in order to take into account the heterogeneity of the TBI patient group and to thoroughly characterise the anatomical lesion pattern after severe TBI. The prospective longitudinal testing of TBI patients with DOC will enable us to follow the progression associated with emerging from unconsciousness (in those patients who recover) and to trace the dynamics of cortico-cortical connectivity indices associated with recovery of consciousness. Candidate connectivity patterns that are expected to promote the re-emergence of consciousness are cortico-cortical connectivity within the default mode network and between prefrontal and parieto-temporal areas. The multimodal assessment will allow the identification of integrated cortico-cortical connectivity indices across brain mapping modalities, which we hypothesise can more reliably predict recovery from DOC as compared with currently used prognostic methods. ${ }^{22}$

\section{METHODS AND ANALYSIS}

\section{Participants and timeline}

We plan to prospectively include a group of 30 patients over the age of 17 in the study. Due to the multimodal nature of our experimental design, a power calculation of the required sample size that would comprise the 
Table 1 Sample size of neuroimaging longitudinal studies in patients in the subacute stage after severe TBI

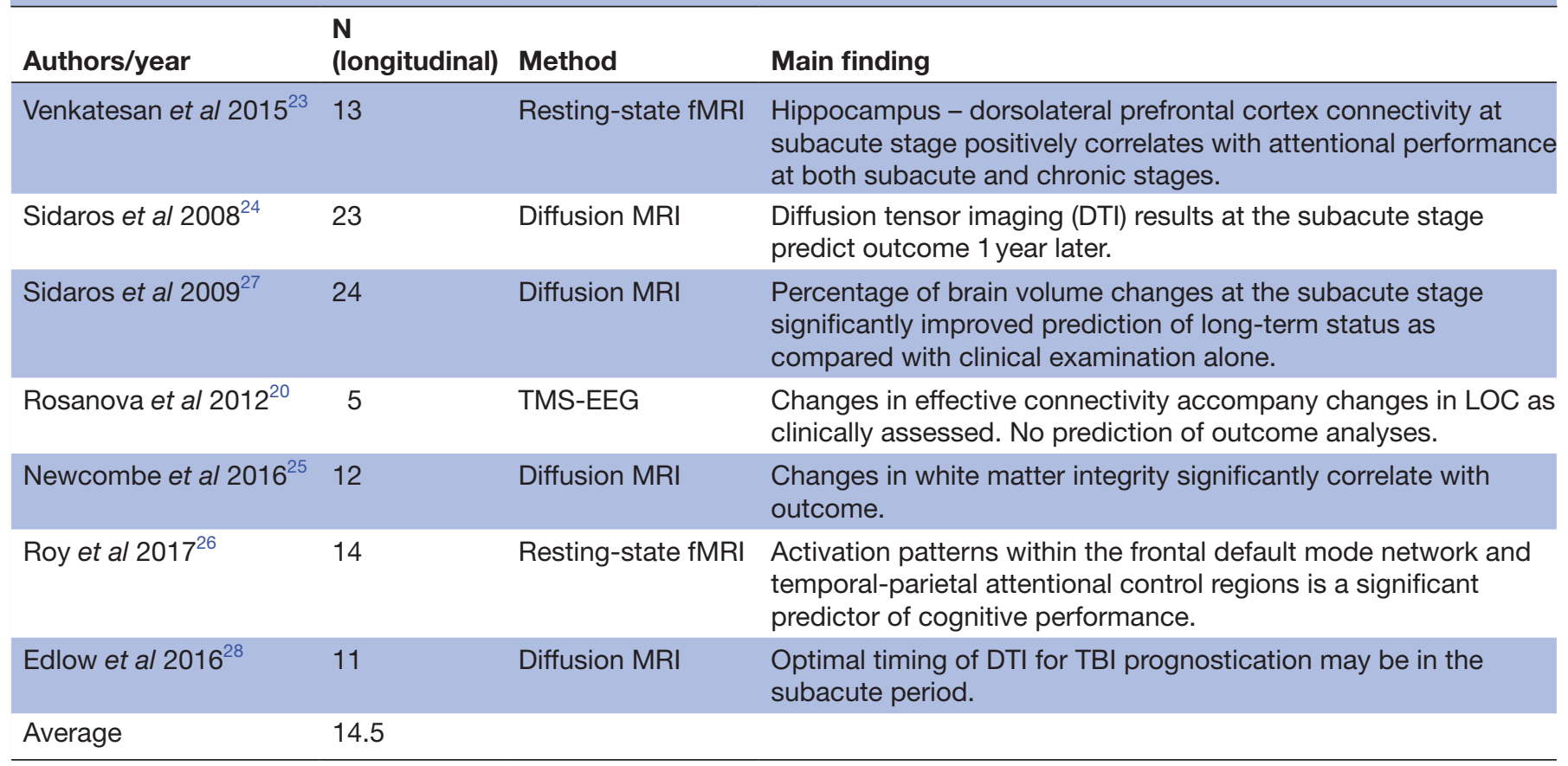

fMRI, functional MRI; LOC, loss of consciousness; TBI, traumatic brain injury; TMS-EEG, transcranial magnetic stimulation with electroencephalography.

combined different methods was not feasible. Following a pragmatic approach, the sample size was then determined based on prior longitudinal studies of connectivity in patients with severe TBI and DOC within the subacute stage where significant results were obtained (see table $\left.1,{ }^{20}{ }^{23-28}\right)$. Studies that had included larger samples examined patients with heterogeneous aetiologies where only a subgroup of patients had traumatic aetiology or had patients in the subacute stage (eg, ref 8$)$, making it difficult to estimate the effect size for this specific patient population. The overall aim of the present sample size definition, thus, is to extend on the average sample size commonly reported (ie, on average about 15 patients per study) in order to account for potential dropouts, deaths and exclusions, as well as to capture the inherent heterogeneity of this patient population. Eligible participants are patients who have suffered closed-head traumatic brain injuries and are subsequently transferred to the Department of Neurorehabilitation, Traumatic Brain Injury at Rigshospitalet (Denmark), located at Hvidovre Hospital. Inclusion in the study and first measurements will take place within the first 2 weeks of admission to the unit, irrespective of the consciousness level at the moment of the inclusion (UWS/VS, MCS or higher). Further measurements will be acquired between 6 and 10 weeks after commencement of rehabilitation, at discharge (approximately 3 months after admission, although it may vary across patients due to clinical progression) and at 1-year follow-up after TBI. Furthermore, a group of 20 gender-matched, age-matched and socioeconomically matched healthy control participants will be included in the study. Healthy participants will be recruited by online and physical ads where they can get in contact with the researcher in charge of recruitment. They will undergo two measurements corresponding to the admission and the discharge time points of the patients, which will be approximately 3 months apart. For a schematic representation of the timeline, see figure 1 . The study protocol is registered within ClinicalTrials.gov with identifier number NCT02424656.

\section{Project workflow}

The project will run over 5 years. The study started in summer 2013 with the recruitment of the first patients and healthy control participants in summer 2014. Recruitment is ongoing, with expected final date for patient recruitment estimated to be winter 2017-2018. Data analysis started in 2014 and will be run parallel to data acquisition until winter 2017-2018. Finally, dissemination of results and article writing will take place between 2017 and 2018. For a schematic representation of the project workflow, see figure 2.

\section{Experimental design}

Each assessment will be divided in three experimental sessions: an MRI session, a session combining TMS with EEG (performed on consecutive days) and a session of FDG-PET. Moreover, clinical evaluation of DOC will be measured by the Coma Recovery Scale-Revised $^{29}$ and the Rancho Los Amigos Scale. ${ }^{30}$ Functional ability will be measured by the Functional Independent Measure $^{31}$, and the Early Functional Abilities Scale ${ }^{32}$ will be performed once a week during admission and at follow-up. 


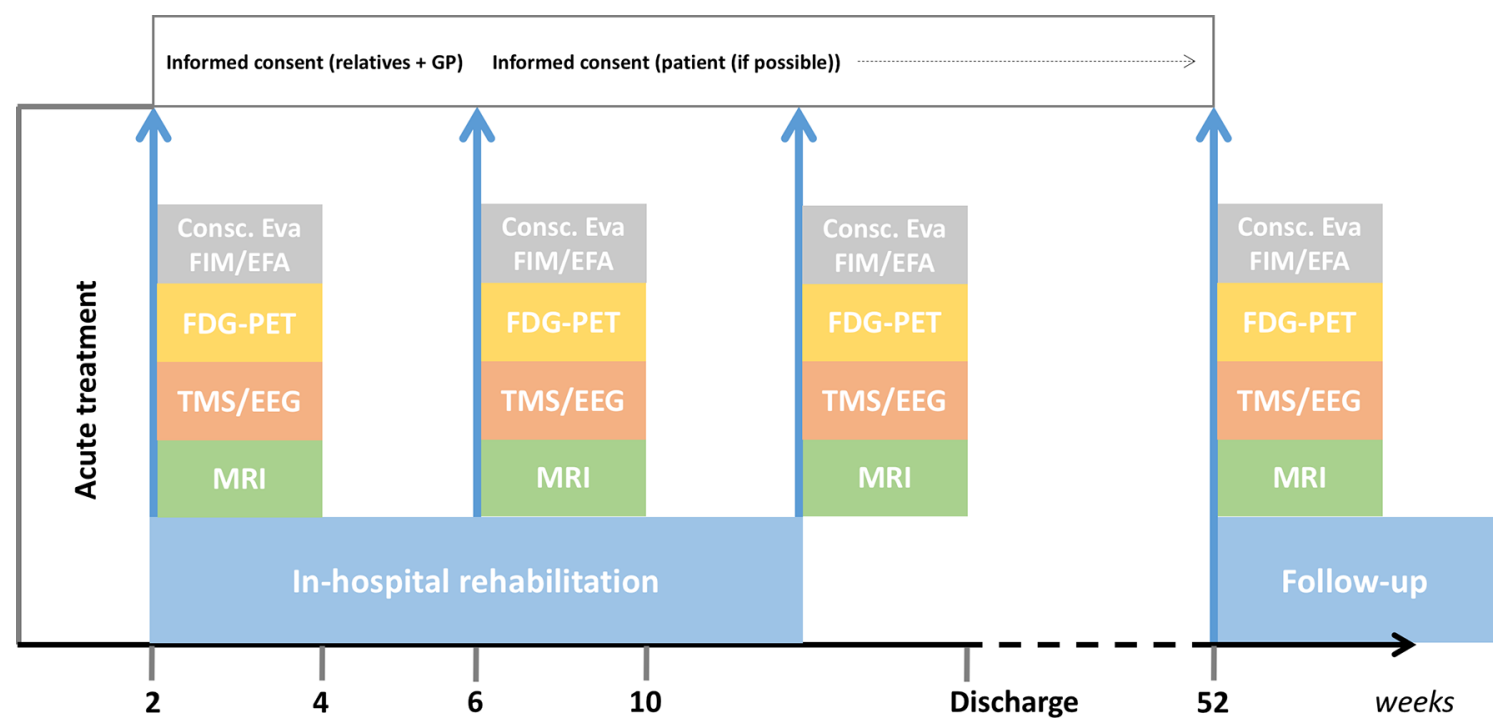

Figure 1 Schematic representation of the timeline for the experimental sessions in relation to the clinical rehabilitation of the patients. Please note that the discharge of the patient does not have a fixed week number but can vary across patients due to clinical reasons. Consc. eva. ('Consciousness evaluation') refers to the clinical evaluation of the consciousness state of the patient via standardised clinical scales. EEG, electroencephalograph; EFA, early functional abilities; FDG-PET, $\left[{ }^{18} \mathrm{~F}\right]-$ fluorodeoxyglucose positron emission tomography; FIM, functional independent measure; MRI, magnetic resonance imaging; TMS, Ttranscranial magnetic stimulation.

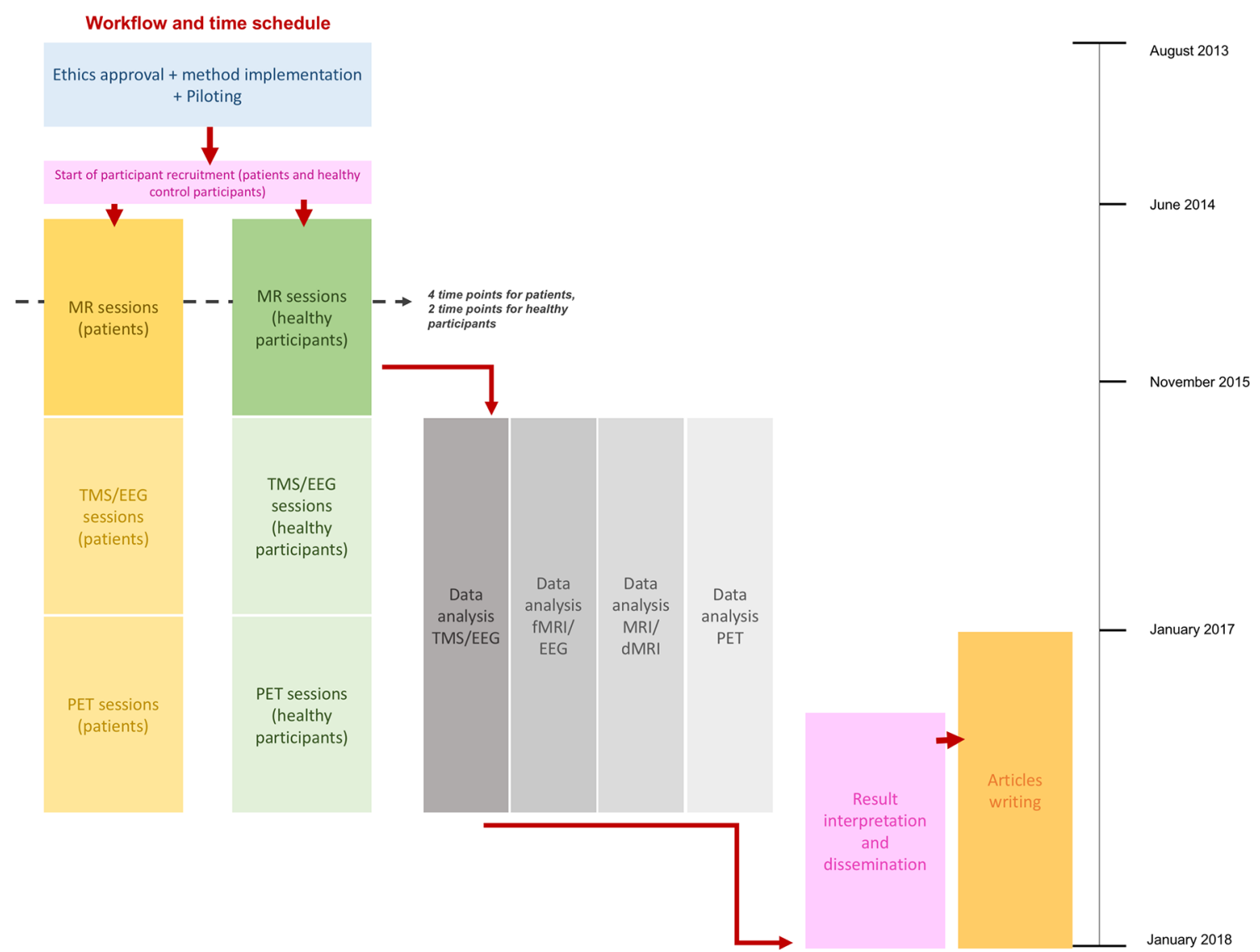

Figure 2 Schematic representation of the workflow. Please notice that some steps overlap due to the requirements of the study (planning on patient recruitment cannot be made in advance since it depends on admission to the rehabilitation department). EEG, electroencephalography; dMRI, diffusion Mangentic resonance imaging; fMRI, functional magnetic resonance imaging; PET, positron emission tomography; MR, Magnetic resonance; TMS, transcranial magnetic stimulation. 
i. Behavioural and clinical assessments. At the time of admission detailed information about demographics, mechanism of injury, neurological deficits, injury severity, acute complications and length of acute treatment will be collected according to a standard protocol. The level of consciousness $^{29} 33$ and length of PTA $^{34}$ will be prospectively assessed with validated scales by trained personnel. Clinical evaluation will include complications (epilepsy, hydrocephalus and pneumonia) and activities of daily living whenever possible.

ii. MRI protocol. Using an echo-planar imaging (EPI) sequence (gradient echo EPI, repetition time $\mathrm{TR}=2150 \mathrm{~ms}$, echo time $\mathrm{TE}=26 \mathrm{~ms}$, GRAPPA acceleration factor 2 , flip angle $78^{\circ}, 42$ axial $3 \mathrm{~mm}$ slices without gap, $192 \mathrm{~mm}$ field of view (FOV), $3 \mathrm{~mm}$ isotropic resolution, 32-channel head coil), whole-brain fMRI will be performed during a $10 \mathrm{~min}$ period of rest with eyes open (resting-state fMRI) (rs-fMRI)) with concurrent EEG (MR-compatible 32-channel EEG cap (EasyCap, Germany) and an online EEG recording system (Brain Vision Recorder, Brain Products, Germany). Due to the differences in brain networks as measured with rs-fMRI between wakefulness and sleep, patients will be maintained awake by a trained nurse using standard clinical procedures. Wholebrain structural images will be acquired to assess macrostructural damage including bleedings. Structural images will comprise of T1-weighted imaging, T2-weighted imaging, fluid-attenuated inversion recovery and susceptibility-weighted imaging, and lesion maps will be delineated for parcellation of each individual brain. Diffusion MRI (dMRI) will cover the whole brain with 61 directions and a $2.3 \mathrm{~mm}^{3}$ isotropic resolution. ${ }^{35} \mathrm{~A}$ $\mathrm{b}$ value of $1000 \mathrm{~s} / \mathrm{mm}^{2}$ will be used for the dMRI sequence from which tractography measures will be derived. A b value of $2000 \mathrm{~s} / \mathrm{mm}^{2}$ and $500 \mathrm{~s} /$ $\mathrm{mm}^{2}$ (with six directions) will be furthermore used for the analysis of Neurite Orientation Dispersion and Density imaging (NODDI), used to derive measures of microstructural white matter integrity. ${ }^{36}$ MRI measurements will be performed on a 3 Tesla whole-body MR Siemens scanner (Magnetom VERIO, Siemens Healthcare, Germany) using a 32-channel head coil. The full scanning session will last approximately 1 hour and $30 \mathrm{~min}$.

iii. EEG with and without concurrent TMS. EEG will be recorded using a TMS-compatible 64-channel EEG system (EasyCap and BrainProducts, Germany). TMS will be applied using a small figure-of-eight coil (50 $\mathrm{mm}$ wing diameter) and a magnetic stimulator (Mag\&More, Germany). Stereotactic neuronavigation (LOCALITE, Germany) will be performed with the individual
T1-weighted MR images of the patients, in order to ensure anatomically sensitive placement of the hotspots and to avoid areas with lesions. The location of the EEG electrodes will be recorded with the neuronavigation system, as an overlay on the three-dimensional-rendered brain surface. The EEG electrode locations will be kept the same across measurements to ensure same spot of cortical stimulation and to minimise the variability of source localisation across sessions. EEG will be recorded during a $10 \mathrm{~min}$ period during which patients will rest with eyes open (resting-state EEG, same procedure as for rs-fMRI) at the beginning and at the end of the experiment. TMS-evoked cortical responses, applied over the posterior parietal and superior frontal cortices, will be recorded according to the protocol described by Rosanova $e t a l .^{20}$ Moreover, a sham condition using concurrent somatosensory and auditory stimulation (as previously done in ref 37 ) will be performed, in order to control for the effects of multisensory stimulation on the EEG-evoked responses.

iv. FDG-PET studies are acquired with Siemens Biograph mCT 128 4R (Siemens Healthcare, Germany). Forty minutes after intravenous injection of $200 \mathrm{MBq}( \pm 10 \%){ }^{18} \mathrm{~F}$-FDG a venous blood sample is drawn to measure plasma glucose level and tracer concentration. Subsequently, a low-dose CT scan (for attenuation correction) followed by a $10 \mathrm{~min}$ PET scan is obtained. An arterial input function will be estimated by scaling a standardised arterial input function ${ }^{38}$ and CMRglc quantified using Sokoloff's autoradiographic method. ${ }^{39}$ All patients will be fasting 6 hours prior to the FDG-PET scan, and exercise is restricted. Due to differences in FDG-PET results between wakefulness and sleep, patients will be maintained awake by a trained nurse using standard clinical procedures for 30 min postinjection while lying in a quiet room with covered eyes.

\section{Data analysis}

Structural MR images will be analysed using statistical parametric mapping software (SPM12 Wellcome Centre of Imaging Neuroscience, Institute of Neurology, UCL, London, UK; http:/ /www.fil.ion.ucl.ac.uk/spm) with the packages for voxel-based cortical thickness and for voxelbased morphometry (Computational Anatomy Toolbox 12). For the rs-fMRI data, graph-theoretical methods will be used for characterising information processing in complex networks. In particular, we will generate functional graphs by correlating mean BOLD signals in parcellated cortical and subcortical regions, using the Desikan-Killiany Atlas implemented in FreeSurfer ${ }^{40}$ and derive measures of network efficiency ${ }^{41}$ for each subject. In addition, we will use group independent component analysis (GIFT software package http://mialab.mrn. 
org/software), to estimate each subject's expression of the default mode network. For the EEG analyses, detrended fluctuation analysis (DFA) for the resting state will be performed in accordance to the workflow set out by Hardstone et $a l^{42}$ DFA measures the degree of longrange temporal correlations in the amplitude envelope in different frequency bands by estimating the so-called scaling exponent, which is expected to increase with increasing level of consciousness due to the hypothesised restoration of cortico-cortical connectivity alongside recovery. ${ }^{20}$ For the TMS-EEG data, in-house scripts as well as Fieldtrip (fieldtrip.fcdonders.nl), running in Matlab (MathWorks), will be used for the analysis of TMS-evoked potentials. TMS-EEG effective connectivity will be analysed according to the methods described in Rosanova et $a l^{20}$ Data analysis of FDG-PET scan for measuring CMRglc will be performed using PMOD software (PMOD Technologies, Zürich, Switzerland). dMRI data will be processed and analysed using the diffusion toolbox of the FSL software package (http://www.fmrib.ox.ac. $\mathrm{uk} / \mathrm{fsl}$ ). Furthermore, a new method called anatomical connectivity mapping (ACM), used to obtain a measure of anatomical connectivity for each voxel in the brain, ${ }^{43}$ will be used. AMICO $^{44}$ (https://github.com/daducci/ AMICO) will be used for the analysis of NODDI. Finally, in order to assess the advantages of obtaining data from multiple modalities in characterisation and prediction of outcomes, we will form multivariate classification and regression models based on multiple modalities. This approach will be based on machine learning and resampling techniques for assessing prediction performance in estimating the clinical evaluation of DOC and recovery from DOC. We will rely on Gaussian process regression, which has as an advantage that regularisation parameters to avoid over-fitting can be identified by a computationally efficient Bayesian learning procedure. This avoids the need for an additional cross-validation procedure to identify regularisation parameters. Due to the relatively low number of data samples, we will perform leave-one-out cross-validation to estimate the unbiased prediction performance. The data dimensionality will be kept low and we will include features from the different modalities, which reflect our prior hypothesis (see below) that the structural and functional long-range connectivity is predictive of level of consciousness.

\section{Expected results}

\section{Overall hypotheses}

The main overarching hypothesis is that residual structural and functional connectivity of long-range cortico-cortical neurons connecting prefrontal-parieto-temporal and cingulate cortices is a major determinant for recovery of consciousness after severe TBI.

Moreover, we hypothesise that the multimodal assessment will enable to identify integrated cortico-cortical connectivity indices across brain mapping modalities, which are associated with recovery from DOC. More specifically, by integrating multiple brain mapping modalities (diffusion and fMRI, online fMRI and EEG, online TMS-EEG, TMS-EEG and diffusion, and TMS-EEG and structural MRI), this study will provide unprecedented insights in how severe TBI alters the brain's 'connectome'. These integrated cortico-cortical connectivity indices may help to predict the probability to recover consciousness and increase prognostic power.

\section{Specific hypotheses}

A set of specific hypotheses will be tested with this project, which will depend on the type of research technique applied:

i. Resting-state EEG and fMRI measurements. Impaired resting-state cortico-cortical connectivity in the 'Default Mode Network' (a set of interconnected areas that show activation during internal tasks such as daydreaming; see, eg, ref45) will be expected, with connectivity of parietal areas being critical for the re-emergence of minimal consciousness. ${ }^{46}$ In addition, we expect that the increase in the level of consciousness is related to an increase in network efficiency as reflected by the average shortest path length. ${ }^{47}$ The restingstate EEG is characterised by oscillations with characteristic fluctuations in frequency and amplitude over time. Amplitude fluctuations of alpha and beta oscillations exhibit long-range temporal correlations that can be investigated using detrended fluctuation analysis $\left(\mathrm{DFA}^{48-51}\right)$. DFA exponents of amplitude fluctuations in the alpha and beta frequency bands of the resting-state EEG are expected to be low ( 0.6) in the lowest levels of consciousness and increase $(>0.7)$ with increasing levels of consciousness. We hypothesise that this reflects increasing levels of complexity of the temporal structure of brain oscillations when recovering from DOC.

ii. Functional cortical reactivity (measured with TMS$E E G)$. Recently, Rosanova and colleagues have shown in 12 chronic TBI patients that TMS-EEG measures of effective connectivity could reliably discriminate between unconscious (UWS) and minimally concious (MCS) patients. ${ }^{20}$ In UWS, focal TMS triggered only a simple, local response, indicating a breakdown of effective connectivity to remote cortical regions as previously demonstrated in the unconscious state of sleep. ${ }^{52}$ In MCS, in contrast, TMS triggered complex activations that sequentially involved remote cortical areas that were more similar to healthy subjects during wakefulness. Longitudinal TMSEEG measurements during gradual recovery of consciousness in a small group of patients $(n=5)$ revealed a re-establishment of long-range cortical connectivity before behavioural correlates of consciousness re-emerged. ${ }^{20}$ Thus, we hypothesise that residual cortico-cortical effective connectivity 
will be revealed as an important neural basis for the recovery of consciousness.

iii. Structural integrity of white-matter pathways (assessed with dMRI). A recent dMRI study showed that structural integrity of white-matter pathways in the default mode network inversely correlated with impairment of consciousness. ${ }^{53}$ Hence, dMRI may help to estimate the potential of the brain to regain consciousness after severe TBI, as this might depend on the extent of axonal damage in long-range cortico-cortical pathways. Thus, we hypothesise that the overall impairment of longrange cortico-cortical connectivity, as assessed with ACM, will be a strong predictor for the recovery of consciousness. ACM is less dependent on the exact location of the structural damage, ${ }^{54}$ as it reflects integrated damage along a tract system, and is therefore particularly well suited when comparing patients with diffuse structural damages.

iv. Cerebral glucose metabolism (assessed with FDG-PET). Improvement of CMRglc within the bilateral frontoparietal network and medial temporoparietal areas (precuneus and Broca's area) at the cortical level, as well as within the thalamus and cingulate cortex at the subcortical level, ${ }^{85}$ during subacute rehabilitation will correlate with increases in the level of consciousness as opposed to a persistent lower level of consciousness. Furthermore, we hypothesise that the degree of reduced CMRglc in the subacute state and at 1-year follow-up is a strong predictor for recovery of consciousness and related to cognitive function.

\section{ETHICS AND DISSEMINATION}

The project has received ethical approval by the local ethics committee of the Capital Region of Denmark no. H-4-2013-186 and by the Danish Data Protection no GLO-2014-49/I-Suite number 03389 (valid until 31 October 2020). A specialised nurse will be present for all the measurements performed on the patients, and a physician will be either present or on call for all the sessions. Any adverse events will be reported to the ethics committee as required by standard protocol. In the early stages of the study, informed consent will be obtained from both the proxy and the patient's general practitioner, a judicial procedure that is required by Danish law. For similar reasons, it is mandatory to obtain informed consent from the included patients as soon as they are capable to reliably perceive and understand information themselves about the purpose of the project, side effects and discomfort associated with the procedures. However, none of the measurements are known to have any adverse impact on either healthy participants or patients with neurological disorders.

In the last 5 years, a range of neuroimaging methods has been successfully used to capture the neural correlates of recovery of consciousness after severe TBI. ${ }^{56}$
Existing data indicate that the residual structural and functional connectivity of long-range cortico-cortical neurons connecting prefrontal-parieto-temporal and cingulate cortices is a major determinant for the recovery of consciousness after severe TBI. While previous brain mapping studies have undoubtedly provided valuable new insights into the neural underpinnings of impaired consciousness in TBI patients, they also have inherent limitations. For example, most studies were carried out in chronic rather than acute or subacute TBI patients with DOC. Those few studies, which have prospectively assessed effective cortico-cortical connectivity, included only very small groups of acute or subacute TBI $(n<10)$ and only used a single functional brain mapping modality in most cases, with recent exceptions. ${ }^{57}$ Moreover, most of the studies did not relate their findings to the underlying changes in structural brain connectivity as measured with dMRI or glucose metabolism as measured with FDG-PET. ${ }^{4}$ Finally, characterisation of patients in terms of the neuroanatomical lesion pattern was usually poor, and the considerable interindividual variation in TBI-induced brain damage was not taken into account when evaluating the link between cortico-cortical connectivity and consciousness. ${ }^{589}$

The present study is highly original in two ways: (1) it will use a unique cohort of patients with TBI, possibly including the biggest sample of TBI patients that has ever been studied prospectively starting in the immediate subacute phase with multiple assessments during the first year of recovery, and (2) it will acquire and integrate connectivity data obtained with a unique combination of cutting-edge brain mapping techniques. Under the main hypothesis of the inherent correlation between multimodal brain imaging data measuring brain functional and neuroanatomical changes in DOC, this novel combination of neuroimaging techniques will dramatically improve the overall picture of the evolution of consciousness in TBI patients. Moreover, it will give a much finer and precise perspective of potential markers predicting the outcome in DOC derived from the combination of multidimensional functional and anatomical consciousness correlates. In addition, the data will shed light on the more basic understanding of the neural mechanisms behind consciousness. This rich multimodal dataset will moreover be used to significantly extend previously established brain connectivity metrics that are associated with the re-emergence of consciousness employing multivariate statistical analysis.

Considering that some degree of disordered consciousness is a cardinal symptom among patients with severe TBI, this comprehensive and prospective examination of how patients regain a conscious state will shed substantial new light on the anatomical and functional underpinnings of re-emergence of consciousness after severe TBI. The high incidence of TBI in Europe calls for an extensive research on the determinants influencing TBI recovery to take place as soon as possible. The recent advancements on multimodal combination of brain stimulation and 
imaging techniques ${ }^{60}$ present the opportunity to gather a much needed overall picture of the neural underpinnings of recovery of consciousness in patients with TBI.

The results will have considerable societal relevance, being highly connected to the neurorehabilitation of patients with TBI and their families and in turn to a better monitoring and prediction of recovery. The results may also lead to valuable neuroimaging biomarkers that can be used to assess the efficacy of consciousness-enhancing therapeutic interventions, for example, new pharmaceutical treatments, and will be reported as original research articles in peer-reviewed journals as well as in international conferences. Publications will follow open-access policies in order to increase the extent of the results outreach. Moreover, group data of brain activation maps will be made available at the public repository NeuroVault (http://www.neurovault.org/). Due to the data protection agreement, as well as ethical consent restrictions, individual data will not be made publicly accessible.

\section{Author affiliations}

${ }^{1}$ Danish Research Centre for Magnetic Resonance, Copenhagen University Hospital Hvidovre, Hvidovre, Denmark

${ }^{2}$ Department of Neurorehabilitation, Traumatic Brain Injury, Copenhagen University Hospital Rigshospitalet, Copenhagen, Denmark

${ }^{3}$ Department of Clinical Physiology and Nuclear Medicine, Centre for Functional and Diagnostic Imaging and Research, Copenhagen University Hospital Hvidovre, Hvidovre, Denmark

${ }^{4}$ Section for Cognitive Systems, DTU Compute, Technical University of Denmark, Copenhagen, Denmark

${ }^{5}$ Department of Neurology, Copenhagen University Hospital Bispebjerg, Copenhagen, Denmark

Contributors VC drafted the first version of the manuscript. VC, SHA, THP, KBL, IP, LPK, HRS, KWA, KHM, KM and IA edited and approved the last version of the manuscript. HRS, IP and LPK designed the first version of the protocol, while VC, THP, KM and KHM worked on add-ons to the original design. VC, THP, KM, KHM and KWA wrote the data analysis section of the manuscript. SHA, LPK, IP, CPH and KBL are responsible for the clinical sections of the manuscript.

Competing interests None declared.

Patient consent The article is a study protocol, and there are no data from patients reported.

Ethics approval Local ethics committee of the Capital Region of Denmark no. H-4-2013-186.

Provenance and peer review Not commissioned; externally peer reviewed.

Data sharing statement No data is reported in the article.

Open Access This is an Open Access article distributed in accordance with the Creative Commons Attribution Non Commercial (CC BY-NC 4.0) license, which permits others to distribute, remix, adapt, build upon this work non-commercially, and license their derivative works on different terms, provided the original work is properly cited and the use is non-commercial. See: http://creativecommons.org/ licenses/by-nc/4.0/

(c) Article author(s) (or their employer(s) unless otherwise stated in the text of the article) 2017. All rights reserved. No commercial use is permitted unless otherwise expressly granted.

\section{REFERENCES}

1. Tosetti P, Hicks RR, Theriault E, et al. Toward an international initiative for traumatic brain injury research. J Neurotrauma 2013;30:1211-22.

2. Blennow K, Brody DL, Kochanek PM, et al. Traumatic brain injuries. Nat Rev Dis Primers 2016;2:16084.
3. Perrin PB, Niemeier JP, Mougeot JL, et al. Measures of injury severity and prediction of acute traumatic brain injury outcomes. $J$ Head Trauma Rehabil 2015;30:136-42.

4. Hannawi Y, Stevens RD. Mapping the Connectome following traumatic brain Injury. Curr Neurol Neurosci Rep 2016;16:44.

5. Owen AM, Coleman MR, Boly M, et al. Detecting awareness in the vegetative state. Science 2006;313:1402.

6. Owen AM, Coleman MR. Using neuroimaging to detect awareness in disorders of consciousness. Funct Neurol 2008;23:189-94.

7. Laureys S, Schiff ND. Coma and consciousness: paradigms (re) framed by neuroimaging. Neuroimage 2012;61:478-91.

8. Stender J, Gosseries O, Bruno MA, et al. Diagnostic precision of PET imaging and functional MRI in disorders of consciousness: a clinical validation study. Lancet 2014;384:514-22.

9. Bruno MA, Majerus S, Boly M, et al. Functional neuroanatomy underlying the clinical subcategorization of minimally conscious state patients. J Neurol 2012;259:1087-98.

10. Thibaut A, Bruno MA, Chatelle $\mathrm{C}$, et al. Metabolic activity in external and internal awareness networks in severely brain-damaged patients. J Rehabil Med 2012;44:487-94.

11. Stender J, Mortensen KN, Thibaut A, et al. The minimal energetic requirement of sustained awareness after brain Injury. Curr Biol 2016;26:1494-9.

12. Giacino JT, Ashwal S, Childs N, et al. The minimally conscious state: definition and diagnostic criteria. Neurology 2002;58:349-53.

13. Bagnato S, Boccagni C, Sant'Angelo A, et al. Longitudinal Assessment of clinical signs of recovery in patients with unresponsive Wakefulness syndrome after traumatic or nontraumatic brain Injury. J Neurotrauma 2017;34:535-9.

14. Anke A, Andelic N, Skandsen T, et al. Functional recovery and Life satisfaction in the First Year after severe traumatic brain Injury: a prospective Multicenter Study of a norwegian National Cohort. $J$ Head Trauma Rehabil 2015;30:E38-49.

15. Whyte J, Gosseries O, Chervoneva I, et al. Predictors of short-term outcome in brain-injured patients with disorders of consciousness. Prog Brain Res 2009;177:63-72.

16. Monti MM, Laureys S, Owen AM. The vegetative state. BMJ 2010;341:c3765.

17. Tononi G. Consciousness as integrated information: a provisional manifesto. Biol Bull 2008;215:216-42.

18. Dehaene S, Changeux JP. Experimental and theoretical approaches to conscious processing. Neuron 2011;70:200-27.

19. Boly M, Massimini M, Tononi G. Theoretical approaches to the diagnosis of altered states of consciousness. Prog Brain Res 2009;177:383-98.

20. Rosanova M, Gosseries O, Casarotto S, et al. Recovery of cortical effective connectivity and recovery of consciousness in vegetative patients. Brain 2012;135(Pt 4):1308-20.

21. Sanders RD, et al. Unresponsiveness not equal unconsciousness. Anesthesiology 2012;116:946-59.

22. Sandsmark DK. Clinical outcomes after traumatic brain Injury. Curr Neurol Neurosci Rep 2016;16:52.

23. Venkatesan UM, Dennis NA, Hillary FG. Chronology and chronicity of altered resting-state functional connectivity after traumatic brain injury. J Neurotrauma 2015;32:252-64.

24. Sidaros A, Engberg AW, Sidaros K, et al. Diffusion tensor imaging during recovery from severe traumatic brain injury and relation to clinical outcome: a longitudinal study. Brain 2008;131(Pt 2):559-72.

25. Newcombe VF, Correia MM, Ledig C, et al. Dynamic changes in White Matter abnormalities correlate with late improvement and deterioration following TBI: a Diffusion Tensor Imaging Study. Neurorehabil Neural Repair 2016;30:49-62.

26. Roy A, Bernier RA, Wang J, et al. The evolution of cost-efficiency in neural networks during recovery from traumatic brain injury. PLoS One 2017;12:e0170541.

27. Sidaros A, Skimminge A, Liptrot MG, et al. Long-term global and regional brain volume changes following severe traumatic brain injury: a longitudinal study with clinical correlates. Neuroimage 2009;44:1-8.

28. Edlow BL, Copen WA, Izzy S, et al. Diffusion tensor imaging in acuteto-subacute traumatic brain injury: a longitudinal analysis. BMC Neurol 2016;16:2.

29. Giacino JT, Kalmar K, Whyte J. The JFK Coma recovery ScaleRevised: measurement characteristics and diagnostic utility. Arch Phys Med Rehabil 2004;85:2020-9.

30. Hagen C, Malkmus D, Durham P. Levels of cognitive function, 1972.

31. Corrigan JD, Smith-Knapp K, Granger CV. Validity of the functional independence measure for persons with traumatic brain injury. Arch Phys Med Rehabil 1997;78:828-34. 
32. Hankemeier A, Rollnik JD. The early functional abilities (EFA) scale to assess neurological and neurosurgical early rehabilitation patients. BMC Neurol 2015;15:207.

33. Malkmus D, Booth B, Kodimer C. Rehabilitation of the head injured adult: comprehensive cognitive management, 1979

34. Levin HS, O'Donnell VM, Grossman RG. The Galveston Orientation and Amnesia Test. A practical scale to assess cognition after head injury. J Nerv Ment Dis 1979;167:675-84.

35. Madsen KS, Baaré WF, Skimminge A, et al. Brain microstructural correlates of visuospatial choice reaction time in children. Neuroimage 2011;58:1090-100.

36. Zhang H, Schneider T, Wheeler-Kingshott CA, et al. NODDI: practical in vivo neurite orientation dispersion and density imaging of the human brain. Neuroimage 2012;61:1000-16.

37. Rossi S, Ferro M, Cincotta M, et al. A real electro-magnetic placebo (REMP) device for sham transcranial magnetic stimulation (TMS). Clin Neurophysiol 2007;118:709-16.

38. Wakita K, Imahori Y, Ido T, et al. Simplification for measuring input function of FDG PET: investigation of 1-point blood sampling method. J Nucl Med 2000;41:1484-90.

39. Sokoloff L, Reivich M, Kennedy C, et al. The [14C]deoxyglucose method for the measurement of local cerebral glucose utilization: theory, procedure, and normal values in the conscious and anesthetized albino rat. $J$ Neurochem 1977;28:897-916.

40. Desikan RS, Ségonne F, Fischl B, et al. An automated labeling system for subdividing the human cerebral cortex on MRI scans into gyral based regions of interest. Neuroimage 2006;31:968-80.

41. Achard S, Bullmore E. Efficiency and cost of economical brain functional networks. PLoS Comput Biol 2007;3:e17.

42. Hardstone R, Poil SS, Schiavone G, et al. Detrended fluctuation analysis: a scale-free view on neuronal oscillations. Front Physiol 2012;3:450.

43. Bozzali M, Parker GJ, Serra L, et al. Anatomical connectivity mapping: a new tool to assess brain disconnection in Alzheimer's disease. Neuroimage 2011;54:2045-51.

44. Daducci A, Canales-Rodríguez EJ, Zhang H, et al. Accelerated Microstructure Imaging via Convex Optimization (AMICO) from diffusion MRI data. Neuroimage 2015;105:32-44.

45. Buckner RL, Andrews-Hanna JR, Schacter DL. The brain's default network: anatomy, function, and relevance to disease. Ann N Y Acad Sci 2008;1124:1-38.
46. Vanhaudenhuyse A, Noirhomme Q, Tshibanda LJ, et al. Default network connectivity reflects the level of consciousness in noncommunicative brain-damaged patients. Brain 2010;133(Pt 1):161-71.

47. Uehara T, Yamasaki T, Okamoto T, et al. Efficiency of a "small-world" brain network depends on consciousness level: a resting-state FMR study. Cereb Cortex 2014;24:1529-39.

48. Linkenkaer-Hansen K, Nikouline VV, Palva JM, et al. Long-range temporal correlations and scaling behavior in human brain oscillations. J Neurosci 2001;21:1370-7.

49. Linkenkaer-Hansen K, Nikulin VV, Palva JM, et al. Stimulus-induced change in long-range temporal correlations and scaling behaviour of sensorimotor oscillations. Eur J Neurosci 2004;19:203-18.

50. Berthouze L, James LM, Farmer SF. Human EEG shows longrange temporal correlations of oscillation amplitude in Theta, Alpha and Beta bands across a wide age range. Clin Neurophysiol 2010;121:1187-97.

51. Berthouze L, Farmer SF. Adaptive time-varying detrended fluctuation analysis. J Neurosci Methods 2012;209:178-88.

52. Massimini M, Ferrarelli F, Huber R, et al. Breakdown of cortical effective connectivity during sleep. Science 2005;309:2228-32.

53. Fernández-Espejo $D$, Soddu A, Cruse D, et al. A role for the default mode network in the bases of disorders of consciousness. Ann Neurol 2012;72:335-43.

54. Lyksborg M, Siebner HR, Sørensen PS, et al. Secondary progressive and relapsing remitting multiple sclerosis leads to motor-related decreased anatomical connectivity. PLoS One 2014;9:e95540.

55. Laureys S. Functional neuroimaging in the vegetative state. NeuroRehabilitation 2004;19:335-41.

56. Boly M, Massimini M, Garrido MI, et al. Brain connectivity in disorders of consciousness. Brain Connect 2012;2:1-10.

57. Annen J, Heine L, Ziegler E, et al. Function-structure connectivity in patients with severe brain injury as measured by MRI-DWI and FDGPET. Hum Brain Mapp 2016;37:3707-20.

58. Gillebert CR, Mantini D. Functional connectivity in the normal and injured brain. Neuroscientist 2013:19:509-22.

59. Wu X, Zou Q, Hu J, et al. Intrinsic Functional Connectivity patterns Predict consciousness level and Recovery Outcome in acquired brain Injury. J Neurosci 2015;35:12932-46.

60. Siebner HR, Bergmann TO, Bestmann S, et al. Consensus paper: combining transcranial stimulation with neuroimaging. Brain Stimul 2009;2:58-80. 